\title{
Clinical correlation of retinal nerve fiber layer thickness in multiple sclerosis patients- A North Indian study
}

\author{
Patel U. ${ }^{1}$, Hilal Ahmad S. ${ }^{2 *}$, Agrawal C. ${ }^{3}$, Rohatgi A. ${ }^{4}$, Grover A. ${ }^{5}$ \\ DOI: https://doi.org/10.17511/ijmrr.2019.i02.03 \\ 1 Upasana Patel, DNB Neurology, Department of Neurology, Sir Gangaram Hospital, New Delhi, India. \\ 2* Sheikh Hilal Ahmad, DNB Neurology, Department of Neurology, Sir Gangaram Hospital, New Delhi, India. \\ 3 C.S. Agrawal, DM Neurology, Department of Neurology, Sir Gangaram Hospital, New Delhi, India. \\ ${ }^{4}$ A. Rohatgi, DM Neurology, Department of Neurology, Sir Gangaram Hospital, New Delhi, India. \\ ${ }^{5}$ A. K. Grover, FRCS, Department of Ophthalmology, Sir Gangaram Hospital, New Delhi, India.
}

Background: Axonal loss is thought to occur early in the course multiple sclerosis (MS) and is supposed to be associated with, and predictive of, neurologic deficits progressing to permanent disability. Axonal loss in the retinal nerve fiber layer (RNFL) is measured by optical coherence tomography (OCT). Material and Methods: A longitudinal observational study, conducted on 30 MS patients. All subjects underwent neurological examination, including expanded disability status scale (EDSS) scoring and OCT on two visits, minimum 2 months apart. Results: Total of 60 eyes of 30 patients were subdivided into 21 eyes having optic neuritis (ON) ['MS - ON'] and 39 eyes without ON. The RNFL thickness (RNFLt) was found to be significantly reduced in all parameters except in temporal quadrant, as the duration of disease increases. Average RNFLt were found to have negative correlation $(r=-0.450)$ with disease duration. Negative correlation $(r=-0.657)$ was also found between EDSS score change and average RNFLt change. The eyes having ON showed statistically significant RNFL thinning as compared to the non - ON fellow eyes. The baseline EDSS score was found to be negatively correlated (moderate degree, $r=-0.348$ ) with baseline average RNFL thickness, with p-value of 0.006 . Conclusions: The RNFLt is not only significantly thinner in those with history of $\mathrm{ON}$, but it is also affected remarkably even in absence of prior ON, suggesting subclinical ongoing axonal loss in patients with MS. The EDSS score is inversely correlated with RNFL thickness.

Keywords: Optical coherence tomography, Retinal nerve fibre layer thickness, Multiple sclerosis, EDSS

Corresponding Author

Sheikh Hilal Ahmad, DNB Neurology, Department of Neurology, Sir Gangaram Hospital, New Delhi, India. Email: drhilal@gmail.com

\section{How to Cite this Article}

Patel U, Ahmad SH, Agrawal CS, Rohatgi A, Grover AK. Clinical correlation of retinal nerve fiber layer thickness in multiple sclerosis patients- A North Indian study. Int J Med Res Rev. 2019;7(2):65-72. Available From

https://ijmrr.medresearch.in/index.php/ijmrr/article/ view/1038
To Browse

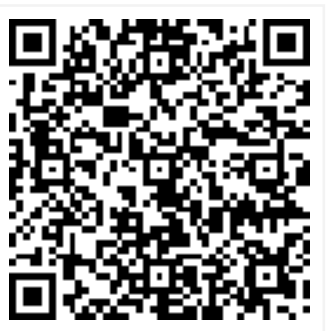

Manuscript Received 2019-02-17

Conflict of Interest No
Review Round 1 2019-02-27

Funding $\mathrm{Nil}$

Review Round 2
2019-03-05
$\begin{gathered}\text { Ethical Approval } \\ \text { Yes }\end{gathered}$

Review Round 2 Yes
Review Round 3

Accepted 2019-03-08

Plagiarism X-checker $5 \%$

(C) 2019 by Upasana Patel, Sheikh Hilal Ahmad, C.S. Agrawal, A. Rohatgi, A. K. Grover and Published by Siddharth Health Research and Social Welfare Society. This is an Open Access article licensed under a Creative Commons Attribution 4.0 International License hittps://creativecommons.org/licenses/by/4.0/ unported [CC BY 4.0]. 


\section{Introduction}

Although multiple sclerosis (MS) has long been considered a primary demyelinating disease, 'axonal loss' is of critical importance since this pathologic change appears to correlate with a patient's ultimate disability [1]. Axonal loss is increasingly thought to occur early in the disease course and is thought to be associated with neurologic deficits and predictive of progression to permanent disability.

Magnetic resonance imaging (MRI) correlates with demyelination related disease severity and prognosticates recurrence; however, it does not identify axonal loss directly and so has a poor correlation with neurological disability. Optical coherence tomography (OCT) is a relatively new technology capable of imaging histologically identifiable layers of the retina in real-time with highly refined resolution, accuracy and reproducibility. Its sensitivity allows direct visualization and measurement of retinal nerve fiber layer thickness (RNFLt) and macular volume with micron-scale resolution [2]. The RNFLt is of particular interest in MS, because optic neuritis (ON) is often a pivotal event in establishing the diagnosis. Moreover, optic nerve dysfunction, which is routinely assessed by optic disc pallor, loss of contrast sensitivity, visual field defects, and delayed latency of visual-evoked potentials (VEPs); may occur subclinically in many other patients [3].

It is estimated that nearly $20 \%$ of all patients with MS present initially with ON, and overall $30-70 \%$ of MS patients develop ON during their disease course $[4,5]$. Preliminary studies have found a loss of RNFLt in ON, likely due to axons that are destroyed in optic nerve $[6,7]$. Axonal loss in the RNFL, as measured by OCT, has the potential to become a model to study axonal loss throughout the central nervous system (CNS) in patients with MS. In this particular study, we intend to correlate RFNL thickness as measured by OCT with EDSS score as a clinical tool for neurological disability in patients of MS in Indian context.

\section{Material and Methods}

Setting and type of study: This was a longitudinal observational study conducted in the Department of Neurology in collaboration with Department of Ophthalmology, in a tertiary care hospital in New Delhi situated in northern India $28.7041^{\circ} \mathrm{N}$, $77.1025^{\circ} \mathrm{E}$.
Sampling methods: For tests of association using bivariate correlations, we assumed a moderate inverse correlation between RFNL thickness and EDSS scores. To detect a moderate correlation ( $r$ $=.30$ ), a sample of 60 analyzable subjects (=eyes, patient no. $=30$ ) would provide $80 \%$ power, to discover that the correlation is significantly different from there being no correlation (i.e. that the correlation would be zero) at the alpha error of 0.05 (one tailed).

Sample collections: For this purpose, 30 consecutive patients who were admitted or reported to our outdoor patient department were included in the study after applying inclusion criteria.

Inclusioncriteria: Adult patients, belonging to either sex, fulfilling Mc Donald's 2010 criteria for MS diagnosis, regardless of MS subtype, previous history of optic neuritis and status of treatment by immuno-modulators; were enrolled as cases.

Exclusion criteria: Subjects with i)Opacities in the ocular media, such as cataracts, corneal haze, vitreous hemorrhage, or inflammatory reaction, ii) Optic nerve abnormalities, glaucoma, retinal disease, and optic disc or peripapillary retinal edema, were excluded.

Ethical considerations: A written informed consent was obtained from all the subjects being enrolled for the study after due approval of the hospital ethics committee. A total of 39 MS patients and 48 relapses were recorded during the study period at our institute. Out of these, 9 had a readmission due to relapse. Among these 39 patients, only 30 were included in the final analyses as rest had uveitis (2), glaucoma (1), and did not provide written consent / were not willing to come for second visit (6).

Patient information was documented in a proforma including presenting complaints, present, past, personal and family history, with details of duration of disease. All patients underwent a detailed physical and neurological examination on two visits 2 months apart. For EDSS scoring, we used the Kurtzke's Functional Systems and Expanded Disability Status Scale. All subjects underwent OCT of both eyes regardless of history of previous optic neuritis and RFNL thickness was measured with characterization in all four quadrants of retina centered at $\mathrm{ONH}$, in department of Ophthalmology; using Stratus OCTTM. 
However, we delayed enrollment in subjects with acute ON for 3 to 6 months to allow the RNFL to stabilize and for at least 1 month after intravenous corticosteroid treatment. [8] Data of EDSS score and RFNL thickness of both eyes as measured by OCT in both visits 2 months apart was compared to find the type of correlation between the two over time; in patients of MS with and without ON.

Statistical analysis: Statistical analysis was performed by the SPSS program for Windows, version 17.0. Continuous variables are presented as mean \pm SD, and categorical variables are presented as absolute numbers and percentage. Data were checked for normality before statistical analysis using Shaipro Wilk test. Normally distributed continuous variables were compared using ANOVA. Multiple comparison testswere used to assess the differences between the individual groups using Bonferroni correction. Categorical variables were analyzed using the chi square test. For all statistical tests, a $p$ value less than 0.05 was taken to indicate a significant difference.

\section{Results}

As per our mandate, 30 cases of multiple sclerosis were enrolled on the basis of revised Mc Donald's criteria (2010); regardless of MS subtype, previous history of ON and status of treatment by immunomodulators. Fifteen patents had history of optic neuritis (MS - ON) and exactly 15 patients had no history of optic neuritis ( MS-NON ). They were studied on two occasions two months apart, the analysis of which is given below. The patients were in the age group ranging from 19 to 50 years. The mean age in 'MS - ON' group was 33.4 years (SD: 8.23) and in 'MS - NON' group was 29.9 years (SD: 10.5).
There was no significant statistical difference between the groups. Female predominance was observed in both the groups, namely 'MS - ON' and 'MS - NON' (11 out of 15 in each group).

The major variables to be compared between 'MS $\mathrm{ON}^{\prime}$ and 'MS -NON' groups included RNFLt in different sectors; namely nasal, temporal, superior\& inferior as well as average RNFLt. The former group was again subdivided into ON - eyes and their fellow non - ON eyes. A total of 60 eyes of 30 patients were subdivided into 21 eyes having ON (of the group 'MS - ON') and 39 eyes without ON.

The latter group also included 9 fellow non - ON eyes of the group 'MS - ON'. The distribution of all patients according to subtype of MS included $60 \%$ of RRMS, $23.33 \%$ of SPMS and $16.67 \%$ of RPMS patients.

The mean disease duration in our study was 3.04 years $(S D=3.305)$ with median of 1.75 years (range 0.2 - 12). The distribution of subjects by disease duration demonstrated a wide spread, with 18 subjects in the 0 to 3 -year duration group, 6 in the 4 to 6 -year group, and 6 in those with more than 6 years duration. The RNFL was found to be significantly thinned in all parameters except in temporal quadrant, as the duration of disease increases

Table 1. Average and inferior RNFLt were found to have moderate negative correlation $(r=-0.450$, -0.395 respectively) with disease duration ( $p$ $<0.001$ and 0.007 respectively) as shown in figure 1. On looking for RNFLt parameters and total number of relapses in patients of MS, RNFLt in all quadrants and average RNFLt was reduced at 2 months (Table 2).

Table-1: Duration of disease and quadrant wise RNFLT thickness (RNFLt).

\begin{tabular}{|l|l|l|l|l|l|}
\hline \multicolumn{2}{|c|}{ RNFL thickness } & \multicolumn{3}{c|}{ Puration of MS } & \multicolumn{2}{l|}{ P value } \\
\cline { 3 - 7 } & & $0-3$ year $(n=18)$ & $4-6$ year $(n=6)$ & $>6$ year $(n=6)$ \\
\hline Nasal & Mean (SD) 0 months & $76.64(17.46)$ & $72.25(19.44)$ & $61.58(15.26)$ & $<0.001$ \\
\hline Temporal & Mean (SD) 0 months & $56.47(8.66)$ & $52.58(15.98)$ & $48.08(14.28)$ & 0.158 \\
\hline Superior & Mean (SD) 0 months & $111.25(16.15)$ & $106.67(10.92)$ & $97.67(8.36)$ & $<0.001$ \\
\hline Inferior & Mean (SD) 0 months & $116.94(11.48)$ & $116.92(20.78)$ & $103.17(10.28)$ & $<0.001$ \\
\hline Average & Mean (SD) 0 months & $90.33(10.67)$ & $87.1(12.84)$ & $77.62(9.23)$ & $<0.001$ \\
\hline
\end{tabular}


Table-2: Number of total relapses and RNFL thickness (RNFLt).

\begin{tabular}{|c|c|c|c|c|c|c|}
\hline \multicolumn{2}{|c|}{ RNFL Thickness } & \multicolumn{4}{|c|}{ Number of relapses } & \multirow[t]{2}{*}{ P value } \\
\hline & & $1(n=8)$ & $2(n=11)$ & $3(n=5)$ & $>4(n=6)$ & \\
\hline \multirow[t]{2}{*}{ RNFL Nasal } & Mean + SD & $66.68+14.54$ & $70.41+14.87$ & $55.2+21.81$ & $55.5+15.04$ & \multirow[t]{2}{*}{0.024} \\
\hline & Min - Max & $34-82$ & $43-105$ & $20-90$ & $34-88$ & \\
\hline \multirow[t]{2}{*}{ RNFL Temporal } & Mean + SD & $52.75+7.46$ & $47.54+6.44$ & $54.2+17.18$ & $37.83+5.54$ & \multirow[t]{2}{*}{$<0.001$} \\
\hline & Min - Max & $41-74$ & $39-60$ & $37-85$ & $30-45$ & \\
\hline \multirow[t]{2}{*}{ RNFL Superior } & Mean + SD & $101.44+16.02$ & $107.64+14.41$ & $89.5+4.57$ & $92+11.85$ & \multirow[t]{2}{*}{0.001} \\
\hline & Min - Max & 50-117 & $68-128$ & $79-96$ & 77-114 & \\
\hline \multirow[t]{2}{*}{ RNFL Inferior } & Mean + SD & $110.56+12.85$ & $107+8.51$ & $104+18.85$ & $94.33+9.02$ & \multirow[t]{2}{*}{0.007} \\
\hline & Min - Max & 78-129 & $91-122$ & $85-140$ & $80-112$ & \\
\hline \multirow[t]{2}{*}{ RNFL Average } & Mean + SD & $82.86+11.35$ & $83.15+8.08$ & $75.72+11.85$ & $69.9+5.87$ & \multirow[t]{2}{*}{0.001} \\
\hline & Min - Max & $51.25-92.75$ & $65.25-97$ & $63.5-102$ & $60.5-78.5$ & \\
\hline
\end{tabular}

Note: RNFLNasal RNFLTemporal, RNFLSuperior, RNFLInferior, RNFLInferior and RNFLAverage are Retinal nerve fibre thickness in nasal, temporal, superior, inferior and average of all four quadrants.

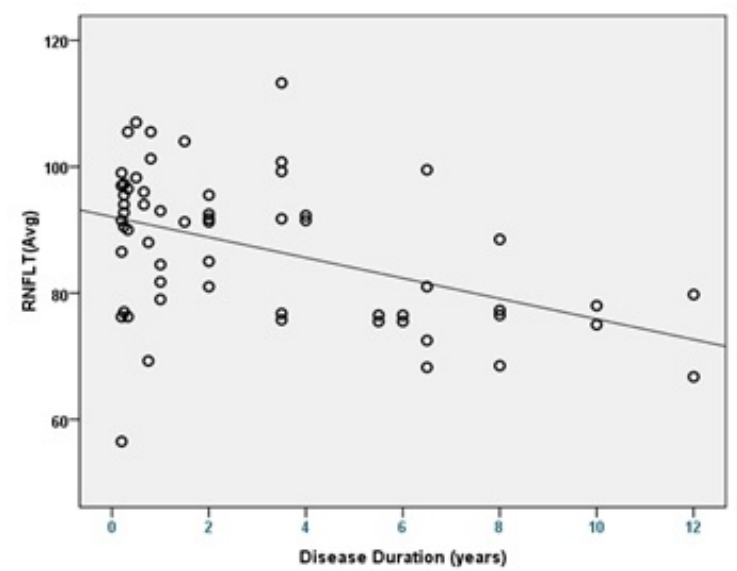

Figure-1: Correlation between disease duration and average RNFL thickness

The eyes having ON showed statistically significant
RNFL thinning in all variables at both the visits as compared to the non - ON fellow eyes of patients with 'MS with ON', the eyes of 'MS without $\mathrm{ON}^{\prime}$ ' and normative data for the respective RNFL value; except the RNFLSuperior at 0 months which is also reduced, but was not found to be statistically significant as show in Table 3.

Even the eyes not having ON also showed thinning in RNFLt as compared to the normative data, suggesting subclinical axonal loss even in absence of history of ON in patients with MS. On post-hoc analysis, multiple subgroup comparisons using Bonferroni correction for each RNFL parameter, where the subgroups were putunder following headings: $1=\mathrm{ON}$ eyes of patients of 'MS - ON', $2=$ fellow non-ON eyes of patients of 'MS - ON', 3=eyes of patients of MS - NON was done. At both the visits, eyes with ON showed significant thinning as compared to other two subgroups. Difference of RNFLA between subgroups 2 and 3, consisting eyes without ON, was not statistically significant; however, both the groups had more thinning as compared to normative data (Table 4).

Table-3: History of optic neuritis and RNFL thickness.

\begin{tabular}{|l|l|l|l|l|l|}
\hline \multirow{2}{*}{ RNFL thickness } & Normative data (Mean + SD) & ON eye $(\mathbf{n = 2 1})$ & Non-ON fellow eye $(\mathbf{n = 9})$ & MS without ON (Patients=15, eyes/n=30) & P value \\
\cline { 3 - 6 } & & O months & 0 months & 0 months & \\
\hline RNFL Nasal & $80.9+18.1$ & $65.09+18.65$ & $81.11+14.39$ & $75.6+17.33$ & 0.038 \\
\hline RNFL Temporal & $69.0+12.7$ & $49.05+9.27$ & $66.11+18.5$ & $53.87+8.4$ & 0.001 \\
\hline RNFL Superior & $124.2+17.9$ & $101.1+18.3$ & $111.67+9.16$ & $110.97+11.93$ & 0.04 \\
\hline RNFL Inferior & $126.1+17.8$ & $109.05+15.65$ & $120.67+16.47$ & $115.83+12.12$ & 0.087 \\
\hline RNFL Average & $107.7+9.9$ & $81.05+11.72$ & $94.89+10.17$ & $89.06+10.47$ & 0.004 \\
\hline
\end{tabular}

Note: RNFLNasal RNFLTemporal, RNFLSuperior, RNFLInferior, RNFLInferior and RNFLAverage are
Retinal nerve fibre thickness in nasal, temporal, superior, inferior and average of all four quadrants. 
Table-4: Sub group comparison of average RNFL thickness and history of optic neuritis.

\begin{tabular}{|l|l|l|l|c|c|}
\hline $\begin{array}{c}\text { Dependent } \\
\text { Variable }\end{array}$ & \multicolumn{1}{|c|}{$\begin{array}{c}\text { (I) } \\
\text { Groups }\end{array}$} & $\begin{array}{c}\text { (J) } \\
\text { Groups }\end{array}$ & $\begin{array}{c}\text { Mean Difference } \\
(\mathbf{I}-\mathrm{J})\end{array}$ & $\begin{array}{c}\text { Std. } \\
\text { Error }\end{array}$ & $\begin{array}{c}\text { P } \\
\text { value }\end{array}$ \\
\hline \multirow{2}{*}{$\begin{array}{l}\text { O months } \\
\text { RNFLAverage }\end{array}$} & 1 & 2 & -13.82937 & 4.33864 & 0.007 \\
\cline { 2 - 6 } & 1 & 3 & -8.00714 & 3.09841 & 0.037 \\
\cline { 2 - 7 } & 2 & 3 & 5.82222 & 4.13880 & 0.495 \\
\hline \multirow{2}{*}{$\begin{array}{l}\text { 2months } \\
\text { RNFLAverage }\end{array}$} & 1 & 2 & -13.78968 & 3.80139 & 0.002 \\
\cline { 2 - 7 } & 1 & 3 & -8.42857 & 2.71474 & 0.009 \\
\cline { 2 - 7 } & 2 & 3 & 5.36111 & 3.62630 & 0.434 \\
\hline
\end{tabular}

( $1=\mathrm{ON}$ eyes of patients of 'MS-ON', $2=$ fellow non$\mathrm{ON}$ eyes of patients of 'MS-ON',3=eyes of patients of MS-NON)

The mean baseline EDSS score at 0 months and 2 months were 3.45(SD: 1.89) and 3.47(SD: 2.25) respectively. The baseline EDSS score was found to be negatively correlated (moderate degree, $r=$ -0.348) with baseline average RNFL thickness, with p-value of 0.006 . So, those having higher EDSS score at baseline were noted to have thinner baseline average RNFL thickness. The moderate degree of negative correlation ( $r=-0.657)$ was also found between EDSS score change and average RNFL thickness change at second visit, which was statistically highly significant $(\mathbf{p}<\mathbf{0 . 0 0 1})$. This suggests that the more the clinical worsening in disease course (increasing EDSS score), the more is the average RNFL thinning (Fig. 2).

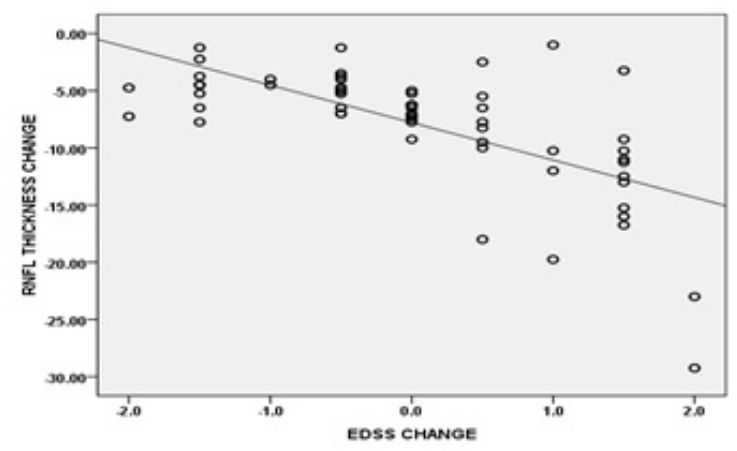

Figure-2: Correlation between change in EDSS

(2 months - 0 months) and average RNFLt (2 months - 0 months)

Table-5: Subtypes of MS and quadrant-wise analysis of RNFL thickness.

\begin{tabular}{|l|l|l|l|l|l|}
\hline \multicolumn{2}{|c|}{ RNFL thickness } & RRMS $(\mathbf{n = 1 8})$ & RPMS $(\mathbf{n = 5})$ & SPMS $(\mathrm{n}=\mathbf{7})$ & \multirow{2}{*}{ Pvalue } \\
\cline { 2 - 5 } \multicolumn{2}{|l|}{} & 2 months & 2 months & 2 months & \\
\hline \multirow{2}{*}{ RNFL Nasal } & Mean(SD) & $68.78(18.29)$ & $59.20(17.4)$ & $51.71(10.37)$ & \multirow{2}{*}{0.079} \\
\cline { 2 - 5 } & Min-Max & $20-96$ & $45-88$ & $34-66$ & \\
\hline
\end{tabular}

\begin{tabular}{|l|l|l|l|l|l|}
\hline \multirow{2}{*}{ RNFL Temporal } & Mean (SD) & $51.39(6.71)$ & $43.20(10.78)$ & $38(5.8)$ & $<$ \\
\cline { 2 - 5 } & Min-Max & $39-65$ & $31-59$ & $30-44$ & \\
\hline \multirow{3}{*}{ RNFL Superior } & Mean (SD) & $105.28(15.01)$ & $91.8(11.03)$ & $88(7.7)$ & \multirow{2}{*}{0.012} \\
\cline { 2 - 6 } & Min-Max & $68-128$ & $79-109$ & $77-98$ & \\
\hline \multirow{2}{*}{ RNFL Inferior } & Mean (SD) & $111.28(8.45)$ & $102.4(20.91)$ & $96.29(8.6)$ & \multirow{2}{*}{0.016} \\
\cline { 2 - 6 } & Min-Max & $97-122$ & $80-133$ & $88-112$ & \\
\hline RNFL Average & Mean (SD) & $84.18(8.75)$ & $74.15(5.79)$ & $68.5(4.26)$ & $<.001$ \\
\cline { 2 - 5 } & Min-Max & $65.25-93.75$ & $66-81.5$ & $60.5-93.75$ & \\
\hline
\end{tabular}

Note: RNFLNasal RNFLTemporal, RNFLSuperior, RNFLInferior, RNFLInferior and RNFLAverage are Retinal nerve fibre thickness in nasal, temporal, superior, inferior and average of all four quadrants.

The RNFLtwas also analyzed in different subtypes of MS. We could not get patients having PPMS (primary progressive MS) during our study period, so rest of the 3 subgroups, i.e. RRMS, RPMS, and SPMS were included and analysed. The trend of RNFL thinning was found towards progressive forms of MS, namely SPMS and RPMS, especially towards the former, in all quadrants and overall RNFL thinning; however, RNFLNasal at 2 months was not statistically significant. The significance level was especially high for RNFLTemporal and RNFLAverage parameters (Table 5).

\section{Discussion}

This pilot study intended to look at the RNFLT as measured by OCT; suggesting a role of OCT as a biomarker for axonal loss in MS patients. The age range of patients included 19 to 50 years, with the mean age in 'MS - ON' group of 33.4 years $(S D=8.23)$ and in 'MS -NON' group of 29.9 years $(S D=10.5)$. There was no significant statistical difference between the groups.

Female predominance $(73.33 \%$ in both the main groups) was found in this study, which was supported by other similar studies carried out in patients with multiple sclerosis which also showed female predominance [9-12]. According to the MS subtype, RRMS, SPMS and RPMS patients constituted $60 \%, 23.33 \%$ and $16.67 \%$ respectively, of total patients. The mean disease duration in our study was 3.04 years $(S D=3.305)$ with median of 1.75 years (range $0.2-12$ ).

The baseline RNFLt was found to be significantly thinned in all parameters (though not statistically significant in temporal quadrant) in those with longer disease duration, and the reduction in RNFLT was even more at second visit in this group $(p<$ 0.001). 
Thus, RNFL thinning was found to be progressively increasing with increasing disease duration. Average and inferior RNFL thickness were found to have moderate degree of negative correlation with disease duration.

Baseline RNFLt in temporal quadrant was shown to be significantly less $(p=0.016)$ in those with four or more relapses, compared to those having $1-3$ relapses, whereas RNFLt at second visit was found to be thinned in all RNFLt quadrants ( $p<0.05)$, again highly significant in temporal quadrant ( $p$ $<0.001)$. Hence, the more the number of relapses, the more is the RNFL thinning, suggesting cumulative axonal loss with each relapse. In studies by Spain et al [10], and Pueyo et al [11], the average RNFLt correlated significantly with MS disease duration which matched with our study. This study also supported the strongest correlation of the inferior quadrant RNFLT with disease duration than any other sector, as reflected in our study also. Similar correlation were also found by Sepulker et al $(r=-0.301, p=0.003)$ and Gencer et al $[12,13]$.

On comparison of the RNFLt parameters; the eyes having ON showed statistically significant RNFL thinning in all variables at both the visits as compared to the Non -ON fellow eyes and MS without ON eyes. Even the MS without ON eyes also showed thinning in RNFLt as compared to the normative data, suggesting subclinical axonal loss even in absence of history of ON in patients with MS. The maximum RNFL thinning was found in the temporal quadrant at both visits in the eyes with ON as compared to their fellow non-ON eyes in the same patients.

This could be because of involvement of the papillomacular bundle (situated on the temporal side of the disc) being affected the most. Pueyo et al11 also showed the greatest thinning in temporal RNFL values in eyes affected with ON, whereas Puliken et al [14], Costello $F$ et al [15] and Saxena et al [12] in addition also showed this pattern of thinning even in the non-affected eyes of MS patients with a trend towards significance. Difference of RNFLA between Non -ON fellow eyesand MS-NON eyes, consisting eyes without ON, was not statistically significant; however, both the groups had more thinning as compared to normative data.

Spain et al with a similar study also demonstrated the existence of a categorical difference between subject cohort-based ON history [10].
They reported mean RNFLA was significantly lower in subjects with a history of ON $(79.06 \mu \mathrm{m}, 95 \% \mathrm{CI}$ 75.31-82.81 $\mu \mathrm{m})$ compared with in those without ON (93.49 $\mu \mathrm{m}, 95 \%$ CI 89.74-97.23 $\mu \mathrm{m} ; p<$ $0.001)$. The distinction in baseline RNFLt based on subject ON history may have implications for using OCT as an outcome measure in populations with MS heterogeneous in ON status.

Other studies also showed a lower RNFLA in the affected eye compared to the unaffected eye in MS patients with ON [8]. Parisi et al [9] examined 14 patients with MS who had one episode of ON. The RNFL thickness was shown to be reduced in ON eyes when compared with unaffected eyes, and control eyes. In another study, Trip et al found a significant reduction in RNFL thickness in ON eyes when compared to both control eyes and unaffected eyes [6]. But in these studies, unaffected eyes' RNFL of MS patients were also found to be significantly thinner than control eyes $[6,8.9]$.

However, no significant differences in the RNFL thickness were found between $\mathrm{ON}$ eyes and unaffected eyes in another study. [12] Fisher's study showed significant thinning of RNFL, 92 micron in MS - NON versus controls 105 micron $(P<0.001)$, suggesting that the unaffected eyes of patients with a history of ON are at a similar risk for axonal loss as the eyes of patients with MS in general [16] In an Indian studyby Singh et al [18] significant RNFL thinning was seen in patients of multiple sclerosis compared to the age and sex matched controls in superior and temporal quadrants of right eye with a $p$ value of 0.002 and in all quadrants in the left eye with a $p$ value of 0.001 .

Significant RNFL thinning was seen in the patients of multiple sclerosis without prior history of optic neuritis than patients with prior history of optic neuritis which was statistically significant with $p$ value of 0.001 [18]. Retrograde trans-synaptic degeneration of retinal ganglion cells due to MS lesions within the posterior optic pathways has been proposed to be involved in RNFL loss in the absence of ON [17]. The mean baseline EDSS score at 0 months and 2 months were 3.45 and 3.47 respectively, with corresponding SD of 1.89 and 2.25, whereas Spain et al had EDSS score with mean + SD of $3.2 \pm 1.6$.

The baseline EDSS score was found to be negatively correlated $(r=-0.348, p=0.006)$ with baseline average RNFLT. 
This matched exactly with the observation by Sepulcre et al, in which RNFL atrophy was correlated with greater disability $(r=-0.348, p=$ 0.001) [12]. An inverse correlation between RNFL thickness and the EDSS was also reported in other studies by Spain et al ( $r s=-0.43, p<0.001)$, Fisher et al $[10,16]$. Thus, those having higher EDSS score at baseline were found to have thinner baseline average RNFLT. The moderate degree of negative correlation ( $r=-0.657)$ was also found between EDSS score change and average RNFL thickness change at 2 months, which was statistically highly significant $(p<0.001)$. This suggests that the more the clinical worsening in disease course (as reflected by increasing EDSS score), the more is the average RNFL thinning.

A two-year longitudinal study of a cohort of $61 \mathrm{MS}$ patients conducted by Sepulcre et al [12] revealed that during follow-up, the subgroup of patients with more active disease developed a thinner temporal quadrant RNFL compared with neurologically stable patients. However, in our study, the comparison between temporal RNFLT and EDSS change between two visits was not found to be statistically significant among the groups, though thinning was present in all 5 groups at 2 months. This may be explained by shorter interval between 2 visits for OCT measurement (i.e. 2 months) in our study, owing to practical limitations. The average RNFL thinning was found to be more in both progressive forms of MS, namely SPMS and RPMS when compared to RRMS, as also reported by Puliken et al [19]. Henderson et al [20] showed that the mean RNFL thickness was significantly reduced in SPMS, but not in PPMS and RNFL loss was most evident in the temporal quadrant, where significant reduction was seen in PPMS versus controls and in SPMS versus PPMS. As we could not enroll PPMS patients, these findings could not be compared with our study. However, our study did show significant temporal quadrant thinning in SPMS patients compared to RRMS.

Clinical outcomes should continue to be the primary endpoints for MS trials, mean while OCT would provide a valuable secondary or tertiary outcome, providing appropriate validation of OCT in relation to clinical and visual outcomes. Measuring RFNLT is a promising tool to assess axonal loss in MS. And it might be time to shift use of OCT as an experimental tool in MS to a clinical tool used to help in management decisions in multiple sclerosis. As of now it's role may be restricted as complimentary to MRI.

\section{Conclusions}

The RNFLt is not only significantly thinner in those with history of ON, but it is also affected remarkably even in absence of prior ON, suggesting subclinical ongoing axonal loss in patients with MS. The EDSS score is inversely correlated with RNFL thickness.

\section{Contribution of Authors}

P.U,S.H.A,A.C.S. conceived and planned the experiments. P.U, A.C.S, R.A, G.A.K, carried out the experiments. P.U, S.H.A, A.C.S and A. Rcontributed to sample preparation. P.U and S.H.A. contributed to the interpretation of the results. S.H. Atook the lead in writing the manuscript. All authors provided critical feedback and helped shape the research, analysis and manuscript.

\section{What this study adds to existing knowledge}

RNFL measurement by OCT can possibly quantify the axonal loss taking place in the visual system in Indian eyes in a manner similar to the Caucasian patients and prognosticate neurological as well as visual outcome. OCT has the potential to become a valuable component of the evaluation of MS patients owing to its quantitative nature, sensitivity, ease of use, availability, low cost (relative to MRI), and noninvasiveness. With further validation and establishment of a relationship with other clinical and MRI measures, OCT can become an appropriate primary or secondary outcome measure.

\section{Acknowledgements}

Mrs Parul for help in statistics.

\section{Reference}

01. De Stefano N, Matthews PM, Antel JP, et al. Chemical pathology of acute demyelinating lesions and its correlation with disability. Ann Neurol. 1995 Dec;38(6)901-9.

DOI: $10.1002 / a n a .410380610$ [Crossref]

02. Sergott RC. Optical coherence tomographymeasuring in-vivo axonal survival and neuroprotection in multiple sclerosis and optic neuritis. Curr Opin Ophthalmol. 2005 Dec;16(6)346-50.

[Crossref] 
03. The clinical profile of optic neuritis. Experience of the Optic Neuritis Treatment Trial, Optic Neuritis Study Group. Arch Ophthalmol. 1991 Dec;109(12)1673-8.

[Crossref]

04. Miller D, Barkhof F, Montalban X, et al. Clinically isolated syndromes suggestive of multiple sclerosis, part I- natural history, pathogenesis, diagnosis, and prognosis. Lancet Neurol. 2005 May;4(5)281-8.

DOI: 10. 1016/ S1474-4422(05)70071-5 [Crossref]

05. Frohman EM, Fujimoto JG, Frohman TC, et al. Optical coherence tomography- a window into the mechanisms of multiple sclerosis. Nat Clin Pract Neurol. 2008 Dec;4(12)664-75.

doi: 10.1038/ncpneuro 0950 [Crossref]

06. Trip SA, Schlottmann PG, Jones SJ, et al. Retinal nerve fiber layer axonal loss and visual dysfunction in optic neuritis. Ann Neurol. 2005 Sep;58(3)383-91.

DOI: 10.1002/ana.20575 [Crossref]

07. Trip SA, Wheeler-Kingshott C, Jones SJ, et al. Optic nerve diffusion tensor imaging in optic neuritis. Neuroimage. 2006 Apr1;30(2)498-505. [Crossref]

08. Costello F, Coupland S, Hodge $W$, et al. Quantifying axonal loss after optic neuritis with optical coherence tomography. Ann Neurol. 2006 Jun;59(6)963-9.

DOI: 10.1002/ana.20851 [Crossref]

09. Parisi V, Manni G, Spadaro M, et al. Correlation between morphological and functional retinal impairment in multiple sclerosis patients. Invest Ophthalmol Vis Sci. 1999 Oct;40(11)2520-7. [Crossref]

10. Spain RI, Maltenfort M, Sergott RC, et al. Thickness of retinal nerve fiber layer correlates with disease duration in parallel with corticospinal tract dysfunction in untreated multiple sclerosis. J Rehabil Res Dev. 2009;46(5)633-42.

[Crossref]

11. Pueyo V, Martin J, Fernandez J, et al. Axonal loss in the retinal nerve fiber layer in patients with multiple sclerosis. MultScler. 2008 Jun;14(5)609-14.

doi: $\quad 10.1177 / 1352458507087326 \quad$ [Crossref]
12. Sepulcre J, Murie-Fernandez $M$, Salinas-Alaman $A$, et al. Diagnostic accuracy of retinal abnormalities in predicting disease activity in MS. Neurology. 2007 May 1;68(18)1488-94. [Crossref]

13. Gencer M, Delipoyraz I, Buttanri B, Turkoglu R, Cetinkaya $Y$, Tireli $H$. Association of Retinal Nerve Fiber Layer Thickness with Disease Disability, Disease Duration, SF-36, and PASAT Scores in Multiple Sclerosis. J Neurol Sci [Turk]. 2013;30(4)702-10.

[Crossref]

14. Pulicken M, Gordon-Lipkin E, Balcer LJ, Frohman E, Cutter G, Calabresi PA. Optical coherence tomography and disease subtype in multiple sclerosis. Neurology. 2007Nov;69(22)2085-92. [Article] [Crossref]

15. Costello F, Hodge W, Pan YI, et al. Tracking retinal nerve fiber layer loss after optic neuritisa prospective study using optical coherence tomography. MultScler. 2008 Aug;14(7)893905.

doi: $\quad 10.1177 / 1352 \quad 458508091367 \quad$ [Crossref]

16. Fisher JB, Jacobs DA, Markowitz $C E$, et al. Relation of visual function to retinal nerve fiber layer thickness in multiple sclerosis. Ophthalmology. 2006 Feb;113(2)324-32. [Crossref]

17. Petzold A, de Boer JF, Schippling S, Vermersch $P$, Kardon $R$, Green $A$, et al. Optical coherence tomography in multiple sclerosis- a systematic review and meta-analysis. Lancet Neurol. 2010;9(9)921-932.

[Article] [Crossref]

18. Singh S, Sharma R, Gurunadh VS, Shankar S. OCT based evaluation of retinal changes in multiple sclerosis. Int $\mathrm{J}$ Res Med Sci. 2017;5;4117-21.

[Article] [Crossref]

19. Pulicken M, Gordon-Lipkin E, Balcer LJ, et al. Optical coherence tomography and disease subtype in multiple sclerosis. Neurology. 2007 Nov 27;69(22)2085-92.

[Crossref]

20. Henderson AP, Trip SA, Schlottmann PG, et al. An investigation of the retinal nerve fibre layer in progressive multiple sclerosis using optical coherence tomography. Brain. 2008 Jan;131(Pt 1) $277-87$. [Crossref] 